\title{
Robustness of metacommunities with omnivory to habitat destruction: disentangling patch fragmentation from patch loss
}

\author{
Jinbao Liao, ${ }^{1,8}$ Daniel Bearup, ${ }^{2,3}$ Yegiao Wang, ${ }^{1}$ Ivan Nijs, ${ }^{4}$ Dries Bonte, ${ }^{5}$ Yuanheng Li, ${ }^{6}$ \\ Ulrich Brose, ${ }^{6,7}$ Shaopeng Wang, ${ }^{6}$ and Bernd Blasius ${ }^{3}$ \\ ${ }^{1}$ Ministry of Education's Key Laboratory of Poyang Lake Wetland and Watershed Research, Jiangxi Normal University, \\ Ziyang Road 99, Nanchang 330022 China \\ ${ }^{2}$ Animal and Plant Sciences, University of Sheffield, Western Bank, Sheffield S10 2TN United Kingdom \\ ${ }^{3}$ Institute for Chemistry and Biology of the Marine Environment (ICBM), University of Oldenburg, \\ Carl-von-Ossietzky-Strasse 9-11, Oldenburg D-26111 Germany \\ ${ }^{4}$ Centre of Excellence Plant and Vegetation Ecology, University of Antwerp (Campus Drie Eiken), \\ Universiteitsplein 1, Wilrijk 2610 Belgium \\ ${ }^{5}$ Department of Biology, Terrestrial Ecology Unit, Ghent University, K. L. Ledeganckstraat 35, Ghent B-9000 Belgium \\ ${ }^{6}$ German Centre for Integrative Biodiversity Research (iDiv) Halle-Jena-Leipzig, Deutscher Platz 5e, Leipzig 04103 Germany \\ ${ }^{7}$ Institute of Ecology, Friedrich Schiller University Jena, Dornburger Strasse 159, Jena 07743 Germany
}

Abstract. Habitat destruction, characterized by patch loss and fragmentation, is a major driving force of species extinction, and understanding its mechanisms has become a central issue in biodiversity conservation. Numerous studies have explored the effect of patch loss on food web dynamics, but ignored the critical role of patch fragmentation. Here we develop an extended patch-dynamic model for a tri-trophic omnivory system with trophic-dependent dispersal in fragmented landscapes. We found that species display different vulnerabilities to both patch loss and fragmentation, depending on their dispersal range and trophic position. The resulting trophic structure varies depending on the degree of habitat loss and fragmentation, due to a tradeoff between bottom-up control on omnivores (dominated by patch loss) and dispersal limitation on intermediate consumers (dominated by patch fragmentation). Overall, we find that omnivory increases system robustness to habitat destruction relative to a simple food chain.

Key words: bottom-up control; food chain; food web robustness; landscape fragmentation; omnivory; patch connectivity; patch-dynamic model; patch loss; species dispersal; top-down control.

\section{INTRODUCTION}

Habitat destruction is one of the most influential factors causing species extinction. Understanding its mechanisms has consequently become a central issue in biodiversity conservation (Thomas and Morris 1995, Tilman and Kareiva 1997, Dieckmann et al. 2000, Haddad et al. 2015). Habitat destruction is characterized by two main processes: patch loss and patch fragmentation (Fahrig 2003), where the latter refers to the division of a continuous habitat into smaller, isolated, sub-habitats. Numerous theoretical and empirical studies have investigated how patch loss and fragmentation separately affect species persistence and therefore community diversity, and significant advances have been made in our understanding of how species respond to them (see review in Fahrig 2002, 2003, Ewers and Didham 2006, Mortelliti et al. 2010). Despite these advances, previous studies generally ignored that species are always embedded in complex food webs in natural ecosystems. How trophic interactions affect the robustness of communities to

Manuscript received 24 February 2017; accepted 15 March 2017. Corresponding Editor: Lewi Stone.

${ }^{8}$ E-mail: jinbaoliao@163.com habitat destruction has thus been largely overlooked (reviews in Pimm and Raven 2000, Holt 2002, Amarasekare 2008, Hagen et al. 2012).

Two decades ago, a few modelling studies based on classical metapopulation theory (Levins 1969, Hanski 1998) began to investigate the impact of patch loss on simple food web dynamics (Bascompte and Solé 1998, Swihart et al. 2001, Melián and Bascompte 2002, Kondoh 2003). These studies consistently concluded that patch loss generally decreases population sizes and disrupts trophic interactions, thereby resulting in increased species loss due to trophic cascading effects (empirically confirmed by Dobson et al. 2006, Cagnolo et al. 2009, Fenoglio et al. 2010, Valladares et al. 2012). Unlike these models, Pillai et al. $(2010,2011,2012)$ developed a novel patch-dynamic model that tracks the patch occupancy of various trophic links instead of individual species, providing a useful framework to study more complex trophic networks undergoing habitat loss. However, it is spatially implicit and thus ignores spatial processes related to patch arrangement.

In reality, many trophic systems are typically heavily fragmented by natural and anthropogenic disturbances, but little is known about how patch fragmentation affects food web dynamics (review in Gonzalez et al. 
2011). According to a single population model, patch connectivity, in contrast to patch fragmentation, can promote species movement range, with higher connectivity providing more colonization opportunities especially for distance-limited dispersers (Liao et al. 2013a, b). In addition, species at different trophic levels usually display different dispersal abilities, with longer-range dispersal typically occurring at higher trophic levels (Peters 1983, McCann et al. 2005, Greenleaf et al. 2007). As a result, differences between the dispersal abilities of predators and their prey are likely to play a key role in modulating food web robustness to landscape fragmentation (Hassell et al. 1993, Amarasekare 2006, 2007).

Among food web studies, omnivory structure, typically defined as feeding on more than one trophic level (Pimm and Lawton 1978), has gained much attention from both theoretical and experimental ecologists (see review by Kratina et al. 2012), as there exists a longstanding debate on the relationship between omnivory and stability. Early theory predicted that adding omnivory to model food webs reduces their stability, suggesting that omnivory should be rare in nature (Pimm and Lawton 1978). Later, detailed observations of food webs revealed that omnivory is widespread in terrestrial, freshwater, marine, and soil ecosystems (Kratina et al. 2012, Digel et al. 2014), and thus omnivory should be both an important species characteristic and a key structural feature of the food webs in which they occur (Stouffer et al. 2012). This led to numerous theoretical investigations showing that, under certain conditions, omnivory is expected to persist and even to confer stability on otherwise unstable food webs (HilleRisLambers et al. 2006, Kratina et al. 2012). For instance, in a spatially implicit context, Pillai et al. (2011) recently found that omnivorous and generalist consumers can support transient increases in food web branching and species richness with habitat loss. Ironically, while the role of omnivory has been explored in typical metapopulations (Melián and Bascompte 2002, Pillai et al. 2011), no work so far has been done on the role of omnivory for foodweb stability in more spatially explicit, and thus more relevant, scenarios.

Based on the modelling framework of Pillai et al. (2010), we develop an extended patch-dynamic model for a tri-trophic system with an omnivorous top predator by explicitly integrating spatial patch arrangement (i.e., patch connectivity) with trophic-dependent dispersal. Specifically, we define that, as the trophic level of a species increases, its dispersal ability also increases from

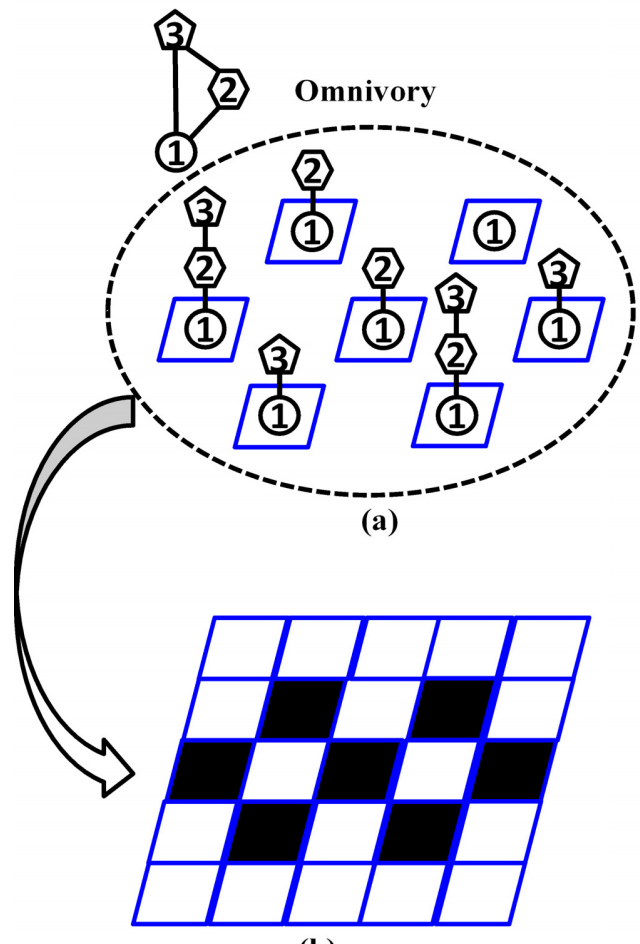

(b)

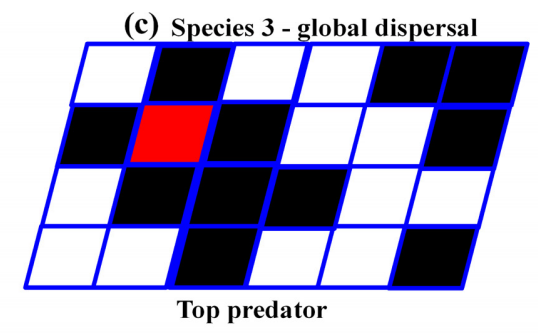

(d) Species 2 - within fragment dispersal

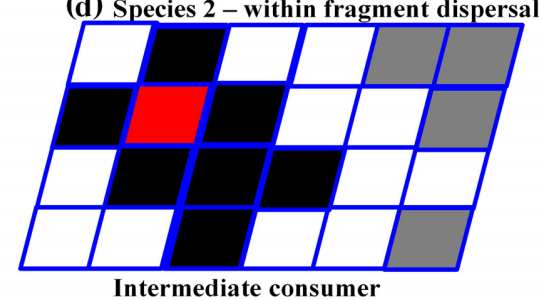

(e) Species 1 - neighbour dispersal

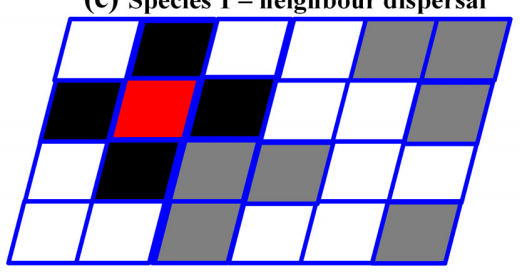

Basal species

FIG. 1. Schematic representation of the model. (a) An omnivorous food web assembled on different food chain configurations in a patch-dynamic framework proposed by Pillai et al. (2010); (b) the patch-dynamic framework embedded into a lattice-structured landscape that consists of suitable (black) and unsuitable (white) patches. (c) An omnivorous species 3 (red) with global dispersal can colonize any suitable patches across the landscape; (d) a consumer species 2 (red) can colonize suitable patches within a connected fragment (using nearest neighbor connections to suitable patches); (e) a basal species 1 with neighbor dispersal (red) can only access to its neighboring suitable patches. In panels c-e, white shows unsuitable patches, gray indicates patches that are suitable but inaccessible due to dispersal range, and black indicates patches that are suitable and accessible within the dispersal range given. 
small-scale nearest neighbor dispersal to global dispersal (illustrated in Fig. 1). Using this model, we explore how community assembles and disassembles in fragmented landscapes and whether omnivory increases system robustness relative to a simple food chain. Robustness is defined as the degree of patch loss and fragmentation that can be tolerated without one or more species going extinct.

\section{Methods \\ Incorporating habitat fragmentation in patch occupancy models}

Patch occupancy models describe the assembly of communities of species on patches of suitable habitat based on colonization and extinction processes (Tilman 1994, Holt 1996, Bascompte and Solé 1998, Hanski 1998, Hanski and Ovaskainen 2000, Melián and Bascompte 2002, Pillai et al. 2010, 2011). Such models are typically spatially implicit, i.e., all patches are equally accessible by any species. However, one of the primary effects of habitat fragmentation, which has been largely overlooked, is to limit the dispersal of species between suitable patches. Thus, in order to investigate the impact of habitat fragmentation on community dynamics, it is necessary to introduce a description of how suitable patches are embedded in the wider landscape and how species disperse between them.

Since patch occupancy models consider only presence or absence of species in a patch (rather than total population), it is natural to regard all patches as being of equal size. With this in mind, the simplest way to introduce spatially explicit structure is to represent the landscape by a two-dimensional square lattice containing two types of patches: suitable habitat $(s)$, which can be colonized, and unsuitable habitat $(u)$, which cannot be colonized and can block distance-limited dispersal (Fig. 1b). Hiebeler (2000, 2007) characterizes such landscapes with two parameters: the density $S$ and clustering degree $q_{s / s}$ of the suitable habitat. The former, density of $s$ habitat, relates intuitively to habitat loss $(U=1-S)$. The latter, clustering degree of $s$ patches, is the probability that a neighbor for a randomly chosen $s$ patch is also suitable. As such, it is proportional to the average size of a habitat fragment, i.e., an area of connected $s$ patches, and thus it provides a measure of habitat fragmentation, defined as $1-q_{s / s}$ (Lloyd 1967, Matsuda et al. 1992, Harada and Iwasa 1994).

This characterization of landscape structure immediately suggests three distinct dispersal modes. The first, global dispersal, allows a species to colonize any $s$ patch in the landscape (Fig. 1c). In typical patch occupancy models, all species are assumed to use this dispersal mode. As such, species of this type are described in our model in exactly the same way as in existing patch occupancy models (Pillai et al. 2010).

The second dispersal mode, within-fragment dispersal, allows dispersal between connected $s$ patches but is blocked by $u$ patches (Fig. 1d). A particular colony of a species that disperses in this way can only colonize a subset of the suitable patches within the landscape, i.e., those in the same habitat fragment. To represent this, we restrict the colonization rate of such species by a factor of $q_{s / s}$, which is proportional to habitat fragment size (Liao et al. 2016a).

The third dispersal mode, nearest neighbor dispersal, allows dispersal only to adjacent suitable patches (Fig. 1e). Thus the number of colonizable patches for such a species is given by the clustering degree of its colonies with unoccupied suitable patches, which can be found using a pair approximation (Appendix S1; Matsuda et al. 1992, Harada and Iwasa 1994, Boots and Sasaki 2000, Liao et al. 2013b, Ying et al. 2014, Liao et al. 2015, 2016a, b).

\section{Model system and analytical approach}

We consider a community of three trophically interacting species: species 1 is the basal species; species 2 consumes species 1; and species 3 consumes both species 1 and 2. The systematic framework proposed by Pillai et al. (2010) was used to determine how trophic interactions between the species would influence their patch occupancy dynamics. However, in addition, we assigned each species one of the dispersal modes described above. In particular, in accordance with observations (Peters 1983, McCann et al. 2005, Greenleaf et al. 2007), we allowed species dispersal range to increase with trophic level, i.e., species 1 with nearest neighbor dispersal, species 2 with dispersal within habitat fragment, and species 3 with global dispersal. The modifications outlined above were applied to the patch-dynamic framework of Pillai et al. (2010) by accounting for species differences in dispersal range (Appendix S1).

Using numerical solutions of this system, we investigate how habitat loss, fragmentation and the combination of both affect species persistence. Additionally we consider how the robustness of the whole community to habitat destruction is affected by the presence of an omnivorous top predator, rather than one that feeds only on species 2 (i.e., a simple food chain; Liao et al. $2016 a$ ). A broad range of biologically reasonable parameter combinations were investigated (Appendix S2: Figs. S1-S4) and found to produce qualitatively consistent community patterns. A representative example is discussed in the following section. Additionally, an important feature of the Pillai et al. (2010) framework is that it individually models the patch dynamics of each trophic link/subcommunity (instead of individual species) that could arise in the system, as predatory species cannot establish on suitable patches without prey species. We make use of this by considering the effect of varying the strength of the trophic interaction between species 3 and species 1 . This allows us to investigate how system robustness varies with the degree of omnivory, e.g., from no prey preference to a strong preference to consume species 2 , displayed by species 3 . 


\section{RESUlts}

We first test how loss of patch availability (i.e., density of patches suitable for colonization) and connectivity (i.e., clustering degree of suitable patches) affect the persistence of a three species community with an omnivorous top predator (Figs. 2, 3). Both forms of habitat destruction reduce species patch occupancy, compromising coexistence of the three species. However, we observe that the relative sensitivity of species 2 and 3 to habitat destruction varies with respect to these two components. In particular, as patch availability decreases, species 3 becomes extinct before species 2 in highly connected landscapes (Figs. 2c, 3a), while this extinction order reverses in more fragmented landscapes (Figs. 2a, b, and 3a). Similarly, as fragmentation increases, species 3 becomes extinct before species 2 at low patch availability, but this switches at high patch availability (Fig. 2e, f). Interestingly, intermediate patch availability and connectivity maximizes the patch occupancy of 1-3 subcommunities (Fig. 3e); this maximum occurs as species 2 becomes extinct (note that species 3 has a feeding preference for species 2).

Next, we compare the robustness of this food web to habitat destruction to that of a simple food chain (Fig. 4). We find that the addition of an omnivorous top predator permits the whole community to tolerate much more patch loss and fragmentation than a food chain, as feeding on different trophic levels promotes the survival of this, usually more vulnerable, species (Fig. 4a-c).
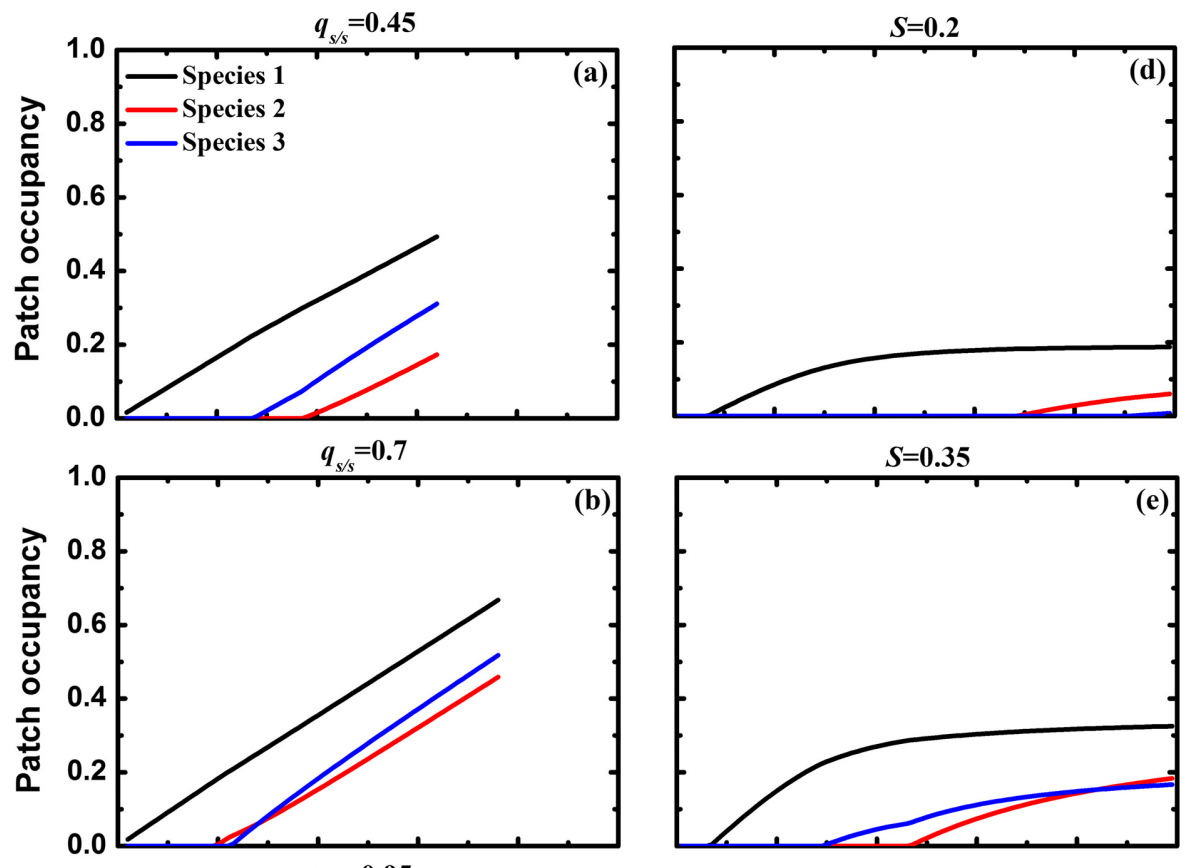

$S=0.5$
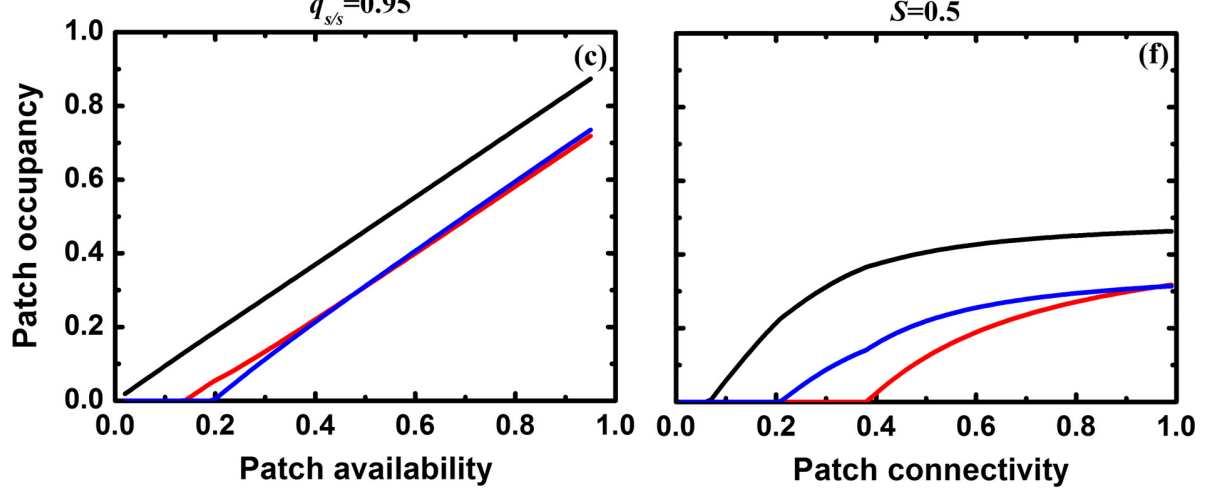

FIG. 2. Individual impacts of patch availability and patch connectivity on species persistence in an omnivorous food web incorporating species dispersal range (species 1, neighbor dispersal; species 2, dispersal within patch clusters; and omnivorous species 3, global dispersal). For the range of patch availability, see Eq. S1 (Appendix S1). Parameter values are as follows: species colonization rate $c_{1}=c_{2}=c_{31}=c_{32}=1$, species intrinsic extinction rate $e_{1}=e_{2}=e_{32}=0.05$, species 3's feeding cost on non-preferred species $e_{31} / e_{32}=3$, and top-down extinction rate $\mu_{32}=\mu_{31}=\mu_{21}=0.025$. $S$, density of suitable habitat; $q_{s / s}$, clustering degree of suitable habitat. 

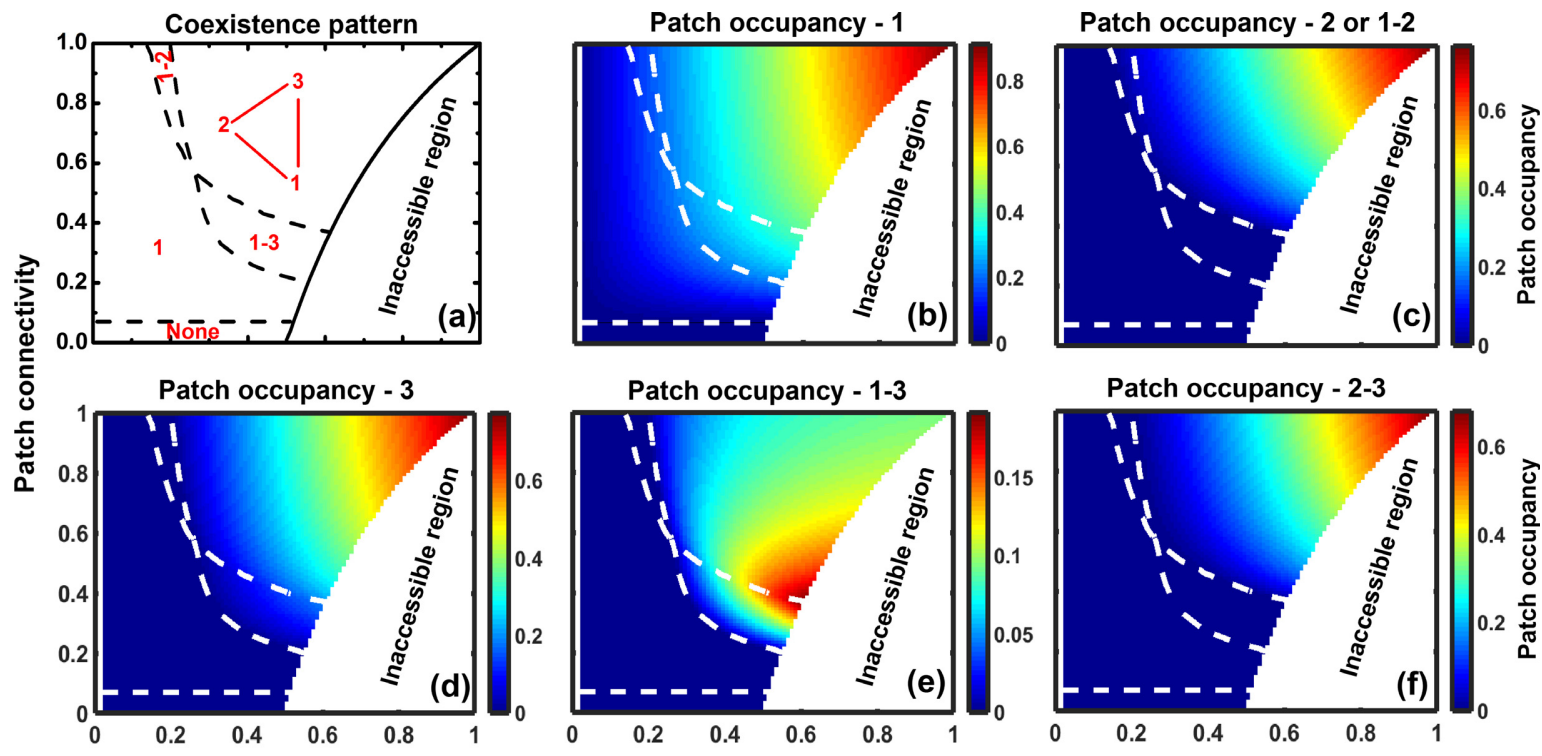

Patch occupancy - 1-3

Patch occupancy - 2-3
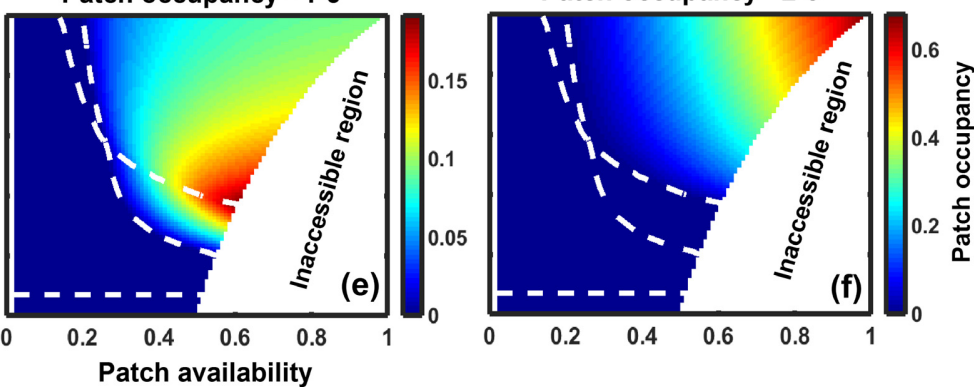

FIG. 3. Interactive effects of patch availability and patch connectivity on spatial patch dynamics of a tri-trophic omnivory food web with longer-range dispersal occurring at higher trophic levels (1, basal species; 2 , intermediate consumer; 3 , omnivorous top predator; and none, extinction of all species). For an explanation of the inaccessible region, see Eq. S1 (Appendix S1). (a) Community composition and pattern (partitioned by dashed lines); (b-f) global patch occupancy of individual species or different trophic links at equilibrium, represented by the rainbow of colors. For parameter values, see Fig. 2.

Varying the parameters describing the degree of omnivory $\left(c_{31}, e_{31}\right.$, and $\left.\mu_{31}\right)$ changes the system robustness (measured by the area of the landscape parameter space where all species coexist). Decreasing the colonization rate of species 3 in $1-3$ subcommunities $\left(c_{31}\right)$ relative to that in the full community $\left(c_{32}\right)$ has a relatively weak effect on system robustness (Fig. 4a), with the strongest effect being a drop in robustness when $c_{31} \leq 0.4 c_{32}$. Increasing the extinction rate of species 3 in $1-3$ subcommunities $\left(e_{31}\right)$ or the predation pressure it exerts on its prey $\left(\mu_{31}\right)$ relative to their counter parts in the full community $\left(e_{32}\right.$ and $\left.\mu_{32}\right)$ results in a large decline in system robustness, approaching the levels found in the simple food chain.

\section{Discussion}

Classical trophically linked non-spatial metacommunity theory only considers models of the relative occurrence of species within patches across the landscape, while ignoring spatial patch arrangement as well as species dispersal range (Holt 1993, 1997, Bascompte and Solé 1998, Melián and Bascompte 2002, Kondoh 2003, Pillai et al. 2010). Based on the framework of Pillai et al. (2010), we developed an extended patch-dynamic model for a tritrophic omnivory system by explicitly linking patch connectivity with species dispersal. The patch dynamics of the species in this model are subject to distinct dispersal modes depending on their trophic position, with longerrange dispersal occurring at higher trophic levels. As such, our spatially extended model allows us to explore the role of landscape fragmentation in mediating food web robustness at the metacommunity scale.

In our simulations, the species display varying sensitivities to patch loss and fragmentation, which strongly depend on their dispersal scale and trophic position in the food web (Figs. 2, 3). In highly connected landscapes habitat loss drives extinctions in trophic-level order (from highest to lowest), in accordance with the trophic rank hypothesis, while in fragmented habitats the intermediate consumer becomes extinct before the omnivore. A similar switch in sensitivity to habitat fragmentation is observed at opposite extremes of habitat loss. This can be explained in terms of the feeding behaviors and dispersal modes we assign to these two species. It is clear that when resources are limited (bottom-up control), the intermediate consumer population is insufficient to support the omnivore (as assumed in the formulation of the trophic rank hypothesis). Consequently, the omnivore competes with the intermediate consumer to prey on the basal species for survival. In highly connected habitats $\left(q_{s / s} \approx 1\right)$, the two species have similar dispersal capabilities and thus the outcome of this competition is determined by the demographic characteristics (i.e., colonization and extinction rates) of these species and the effect of intraguild predation of the omnivore on the intermediate consumer. Since we assume that the omnivore prefers to feed on species 2, it typically has a competitive disadvantage when feeding on species 1 and thus will become extinct first. In contrast, in fragmented habitats, the colonization rate of the intermediate consumer is reduced because of its dispersal limitation. For 

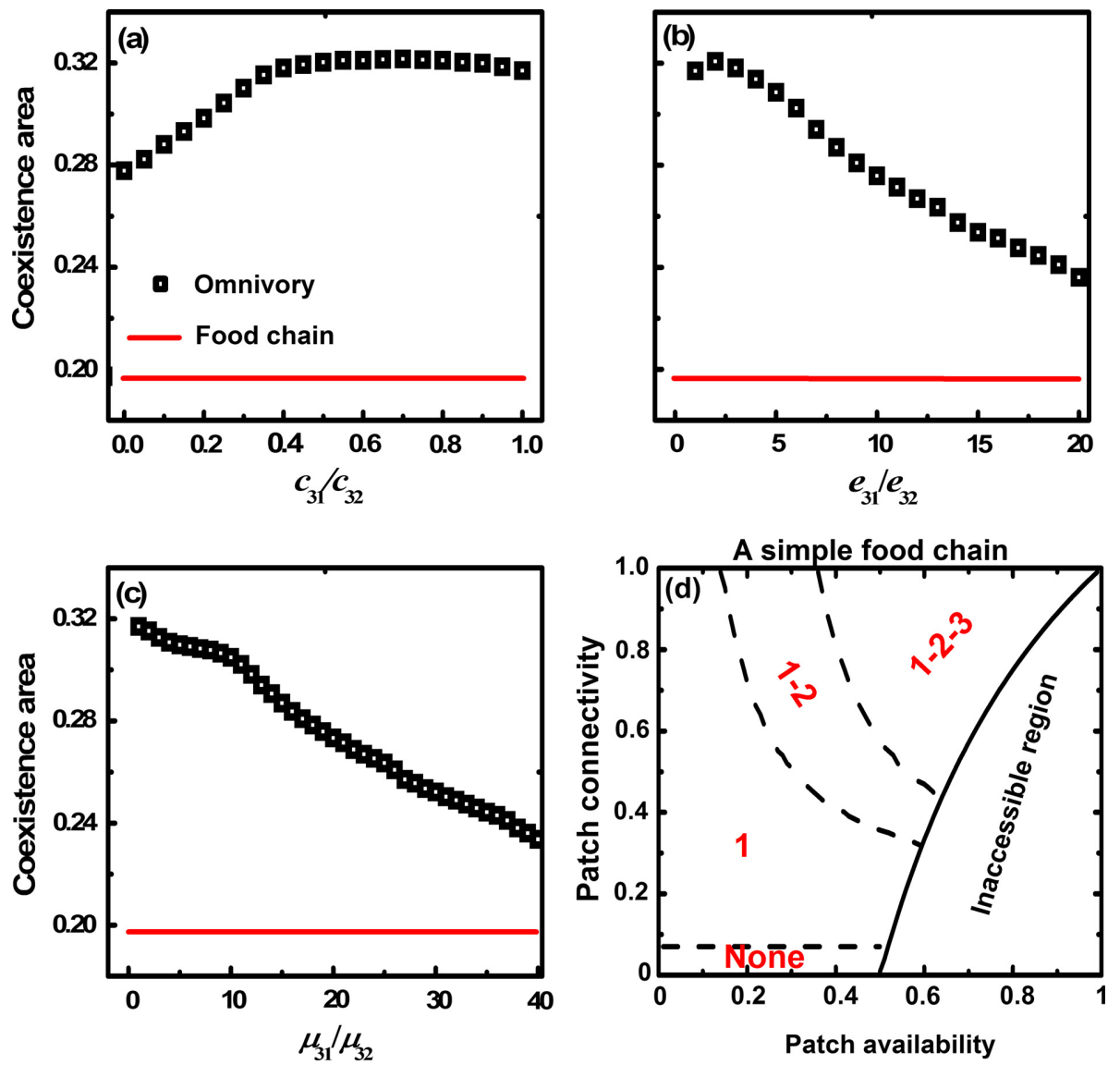

FIG. 4. Impact of variation in the trophic interaction between species 1 and 3 (by varying $c_{31}, e_{31}$, and $\mu_{31}$ ) on the range of landscapes types where all species can coexist for the food web with omnivory $\square$, relative to a simple food chain with $1 \rightarrow 2 \rightarrow 3$ link. $(a-c)$ Area of the region of patch-loss-habitat-fragmentation space where all species coexist (red line shows the area of this region for a food chain); (d) community pattern (partitioned by dashed lines) of a food chain at different levels of habitat destruction (none, extinction of all species). For an explanation of the inaccessible region: see Eq. S1 (Appendix S1). For other parameter values, see Fig. 2.

sufficiently fragmented habitats, this switches the competitive disadvantage to the intermediate consumer resulting in it becoming extinct first.

Most previous spatially implicit modelling studies have focused on the persistence of the intermediate consumer in the omnivory system, because the interplay of competition for basal species and predation by the omnivore confers the highest extinction likelihood to the consumer. However, the inclusion of spatial heterogeneity in our model introduces a more complex tradeoff between these trophic interactions and the dispersal abilities of the consumer and omnivore. In particular, whether the consumer is the most vulnerable species depends on habitat structure and the degree of omnivory, that is, the relative cost to the omnivore of feeding on the basal species instead of the intermediate consumer. In our model, high vulnerability of consumer occurs only at both high fragmentation and omnivory degree (Appendix S2: Fig. S4). Despite this, the region where all species can survive is always larger for the food web with an omnivorous top predator relative to the simple food chain regardless of its omnivory degree (Fig. 4; Appendix S2: Fig. S4), indicating that introducing an omnivore increases system robustness to habitat destruction.

This effect of omnivores on food web robustness has previously been observed in empirical studies (review in Kratina et al. 2012). Our model provides an insight into a potential mechanism that may be responsible for this phenomenon. Habitat destruction eventually limits system resources to the point that the intermediate consumer population cannot support the top predator in a food chain. However, under the same conditions, the omnivore is able to survive by consuming the basal species (e.g., an adaptive feeding strategy cf. Kratina et al. [2012]). While this places it in competition with the intermediate consumer, these species can coexist due to the tradeoff between competitive advantage (for the intermediate consumer) and dispersal advantage (for the omnivore). This allows the full community to survive at higher 
levels of habitat destruction. It is worth mentioning that, in the same review, Kratina et al. (2012) proposed habitat heterogeneity as a critical factor for trophic system robustness, although they focused on prey rather than omnivore refugia as we do here.

Note that this effect of omnivory is strongly dependent on the strength and profitability of the trophic interaction with the basal species. In particular, if the colonization rate of the omnivore population is reduced when feeding on the basal species, or the extinction rate of either omnivore or basal species in such communities is increased, the robustness of the overall system decreases. These changes limit the maximum growth rate of the omnivore population when feeding on the basal species, reducing the benefit obtained from this interaction. As a result, the robustness of the food web with an omnivore declines towards that of a simple food chain.

Our modelling predictions could be further validated by both microcosm experiments and field observations. Controlled microcosms have already been used to investigate how omnivory alters food web stability and persistence (Lawler and Morin 1993, Morin and Lawler 1996, Diehl and Feissel 2000, Liess and Diehl 2006). By incorporating the methods of Staddon et al. (2010) and Chisholm et al. (2011) to produce fragmented habitats, it would be possible to compare the robustness of tri-trophic communities with and without an omnivorous top predator directly to corroborate our predictions concerning the increased stability conferred by omnivory. Larger-scale observations can also provide evidence for effects of omnivory on food web dynamics, e.g., Fagan (1997) tested food web stability as a function of an increasing degree of omnivory in natural arthropod assemblages, and Long et al. (2011) observed omnivory at the predator level in a subtidal marine food web. However, in this context, we argue that it is most appropriate to look for general trends in the relative robustness of these two food web modules rather than seeking specific agreement with our model predictions.

In conclusion, using an extended patch-dynamic model for a tri-trophic community containing an omnivorous top-predator, we find that the sensitivity of species to habitat destruction depends on the relative degree of habitat loss and fragmentation, their trophic position and dispersal characteristics. In particular, differing landscape configurations favor one or other of the intermediate consumer and the omnivore. Finally, and most fundamentally, we find that the tri-trophic system with an omnivorous top predator is more robust to landscape fragmentation than a simple food chain, since its ability to feed on the basal species allows it to behave as a second consumer. Its superior dispersal range then permits it to find refugia that the true intermediate consumer, which is a superior competitor but a poor disperser, cannot invade.

\section{ACKNOWLEDGments}

This study was supported by the DFG through grant FOR 1748 , the Ministry of Science and Culture of Lower Saxony in the project "Biodiversity-Ecosystem Functioning across marine and terrestrial ecosystems," and the Opening Fund of Key Laboratory of Poyang Lake Wetland and Watershed Research (Jiangxi Normal University), Ministry of Education (No. PK2015004 and No. TK2016002). Specific author contributions to this paper were as follows: J. Liao, D. Bearup, and B. Blasius conceived the study and constructed the model; J. Liao performed the analysis of the model; J. Liao and D. Bearup wrote the manuscript with significant input from the other authors.

\section{Literature Cited}

Amarasekare, P. 2006. Productivity, dispersal and the coexistence of intraguild predators and prey. Journal of Theoretical Biology 243:121-133.

Amarasekare, P. 2007. Spatial dynamics of communities with intraguild predation: The role of dispersal strategies. American Naturalist 170:819-831.

Amarasekare, P. 2008. Spatial dynamics of food webs. Annual Review of Ecology, Evolution, and Systematics 39:479-500.

Bascompte, J., and R. V. Solé. 1998. Effects of habitat destruction in a prey-predator metapopulation model. Journal of Theoretical Biology 195:383-393.

Boots, M., and A. Sasaki. 2000. The evolutionary dynamics of local infection and global reproduction in host-parasite interactions. Ecology Letters 3:181-185.

Cagnolo, L., G. Valladares, A. Salvo, M. Cabido, and M. Zak. 2009. Habitat fragmentation and species loss across three interacting trophic levels: Effects of life-history and food web traits. Conservation Biology 23:1167-1175.

Chisholm, C., Z. Lindo, and A. Gonzalez. 2011. Metacommunity diversity depends on connectivity and patch arrangement in heterogeneous habitat networks. Ecography 34:415-424.

Dieckmann, U., R. Law, and J. A. J. Metz. 2000. The geometry of ecological interactions: simplifying spatial complexity. Cambridge University Press, Cambridge, UK.

Diehl, S., and M. Feissel. 2000. Effects of enrichment on threelevel food chains with omnivory. American Naturalist 155:200-218.

Digel, C., A. Curtsdotter, J. Riede, B. Klarner, and U. Brose. 2014. Unravelling the complex structure of forest soil food webs: higher omnivory and more trophic levels. Oikos 123:1157-1172.

Dobson, A., et al. 2006. Habitat loss, trophic collapse, and the decline of ecosystem services. Ecology 87:1915-1924.

Ewers, R. M., and R. K. Didham. 2006. Confounding factors in the detection of species responses to habitat fragmentation. Biological Reviews 81:117-142.

Fagan, W. F. 1997. Omnivory as a stabilizing feature of natural communities. American Naturalist 150:554-567.

Fahrig, L. 2002. Effect of habitat fragmentation on the extinction threshold: a synthesis. Ecological Applications 12:346-353.

Fahrig, L. 2003. Effects of habitat fragmentation on biodiversity. Annual Review of Ecology, Evolution, and Systematics 34:487-515.

Fenoglio, M. S., A. Salvo, M. Videla, and G. R. Valladares. 2010. Plant patch structure modifies parasitoid assemblage richness of a specialist herbivore. Ecological Entomology 35:594-601.

Gonzalez, A., B. Rayfield, and Z. Lindo. 2011. The disentangled bank: How loss of habitat fragments and disassembles 
ecological networks. American Journal of Botany 98:503516.

Greenleaf, S. S., N. M. Williams, R. Winfree, and C. Kremen. 2007. Bee foraging ranges and their relationship to body size. Oecologia 153:589-596.

Haddad, N. M., et al. 2015. Habitat fragmentation and its lasting impact on Earth's ecosystems. Science Advances 1: e1500052.

Hagen, M., et al. 2012. Biodiversity, species interactions and ecological networks in a fragmented world. Advances in Ecological Research 46:89-210.

Hanski, I. 1998. Metapopulation dynamics. Nature 396:41-49.

Hanski, I., and O. Ovaskainen. 2000. The metapopulation capacity of a fragmented landscape. Nature 404:755-758.

Harada, Y., and Y. Iwasa. 1994. Lattice population dynamics for plants with dispersing seeds and vegetative propagation. Research on Population Ecology 36:237-249.

Hassell, M. P., H. C. J. Godfray, H. N. Comins, P. M. Kareiva, J. G. Kingsolver, and R. B. Huey. 1993. Effects of global change on the dynamics of insect host-parasitoid interactions. Biotic Interactions and Global Change 402-423.

Hiebeler, D. 2000. Populations on fragmented landscapes with spatially structured heterogeneities: landscape generation and local dispersal. Ecology 81:1629-1641.

Hiebeler, D. 2007. Competing populations on fragmented landscapes with spatially structured heterogeneities: improved landscape generation and mixed dispersal strategies. Journal of Mathematical Biology 54:337-356.

HilleRisLambers, R., J. Van De Koppel, and P. M. Herman. 2006. Persistence despite omnivory: benthic communities and the discrepancy between theory and observation. Oikos 113: 23-32.

Holt, R. D. 1993. Ecology at the mesoscale: the influence of regional processes on local communities. Pages $77-88$ in R. E. Ricklefs and D. Schluter, editors. Species diversity in ecological communities: historical and geographical perspectives. University of Chicago Press, Chicago, Illinois, USA.

Holt, R. D. 1996. Food webs in space: an island perspective. Pages 313-323 in G. A. Polis and K. O. Winemiller, editors. Food webs. Chapman \& Hall, New York, New York, USA.

Holt, R. D. 1997. Community modules. Pages 333-349 in A. C. Gange and V. M. Brown, editors. Multi-trophic interactions in terrestrial ecosystems. Blackwell, Oxford, UK.

Holt, R. D. 2002. Food webs in space: on the interplay of dynamic instability and spatial processes. Ecological Research 17:261-273.

Kondoh, M. 2003. Habitat fragmentation resulting in overgrazing by herbivores. Journal of Theoretical Biology 225:453-460.

Kratina, P., R. M. Lecraw, T. Ingram, and B. R. Anholt. 2012. Stability and persistence of food webs with omnivory: Is there a general pattern? Ecosphere 3:794-804.

Lawler, S. P., and P. J. Morin. 1993. Food-web architecture and population-dynamics in laboratory microcosms of protists. American Naturalist 141:675-686.

Levins, R. 1969. Some demographic and genetic consequences of environmental heterogeneity for biological control. Bulletin of the Entomological Society of America 15:237-240.

Liao, J., J. Chen, Z. Ying, D. E. Hiebeler, and I. Nijs. 2016a. An extended patch-dynamic framework for food chains in fragmented landscapes. Scientific Reports 6:33100.

Liao, J., Z. Li, D. E. Hiebeler, M. EI-Bana, G. Deckmyn, and I. Nijs. 2013a. Modelling plant population size and extinction thresholds from habitat loss and fragmentation: effects of neighbouring competition and dispersal strategy. Ecological Modelling 268:9-17.

Liao, J., Z. Li, D. E. Hiebeler, Y. Iwasa, J. Bogaert, and I. Nijs. 2013b. Species persistence in landscapes with spatial variation in habitat quality: a pair approximation model. Journal of Theoretical Biology 335:22-30.

Liao, J., Z. Ying, D. E. Hiebeler, Y. Wang, T. Takada, and I. Nijs. 2015. Species extinction thresholds in the face of spatially correlated periodic disturbance. Scientific Reports 5: e15455.

Liao, J., Z. Ying, D. A. Woolnough, A. D. Miller, Z. Li, and I. Nijs. 2016b. Coexistence of species with different dispersal across landscapes: a critical role of spatial correlation in disturbance. Proceedings of the Royal Society B 283: 20160537.

Liess, A., and S. Diehl. 2006. Effects of enrichment on protist abundances and bacterial composition in simple microbial communities. Oikos 114:15-26.

Lloyd, M. 1967. Mean crowding. Journal of Animal Ecology 36:1-30.

Long, Z. T., J. F. Bruno, and J. E. Duffy. 2011. Food chain length and omnivory determine the stability of a marine subtidal food web. Journal of Animal Ecology 80:586-594.

Matsuda, H., N. Ogita, A. Sasaki, and K. Sato. 1992. Statistical mechanics of population: the lattice Lotka-Volterra model. Progress in Theoretical Physics 88:1035-1049.

McCann, K. S., J. R. Rasmussen, and J. Umbanhowar. 2005. The dynamics of spatially coupled food webs. Ecology Letters 8:513-523.

Melián, C. J., and J. Bascompte. 2002. Food web structure and habitat loss. Ecology Letters 5:37-46.

Morin, P. J., and S. P. Lawler. 1996. Effects of food chain length and omnivory on population dynamics in experimental food webs. Pages 218-230 in G. A. Polis, and K. O. Winemiller, editors. Food webs: integration of patterns and dynamics. Chapman and Hall, New York, New York, USA.

Mortelliti, A., G. Amori, and L. Boitani. 2010. The role of habitat quality in fragmented landscapes: a conceptual overview and prospectus for future research. Oecologia 163: 535-547.

Peters, R. H. 1983. The ecological implications of body size. Cambridge University Press, Cambridge, UK

Pillai, P., M. Loreau, and A. Gonzalez. 2010. A patch-dynamic framework for food web metacommunities. Theoretical Ecology 3:223-237.

Pillai, P., A. Gonzalez, and M. Loreau. 2011. Metacommunity theory explains the emergence of food web complexity. Proceedings of the National Academy of Sciences USA 108:19293-19298.

Pillai, P., A. Gonzalez, and M. Loreau. 2012. Evolution of dispersal in a predator-prey metacommunity. American Naturalist 179:204-216.

Pimm, S. L., and J. H. Lawton. 1978. Feeding on more than one trophic level. Nature 275:542-544.

Pimm, S. L., and P. Raven. 2000. Biodiversity: extinction by numbers. Nature 403:843-845.

Staddon, P., Z. Lindo, P. D. Crittenden, F. Gilbert, and A. Gonzalez. 2010. Connectivity, non-random extinction and ecosystem function in experimental metacommunities. Ecology Letters 13:543-552.

Stouffer, D. B., M. Sales-Pardo, M. I. Sirer, and J. Bascompte. 2012. Evolutionary conservation of species' roles in food webs. Science 335:1489-1492.

Swihart, R. K., Z. Feng, N. A. Slade, D. M. Mason, and T. M. Gehring. 2001. Effects of habitat destruction and resource supplementation in a predator-prey metapopulation model. Journal of Theoretical Biology 210:287-303.

Thomas, J. A., and M. G. Morris. 1995. Rates and patterns of extinction among British invertebrates. Pages 111-130 in J. H. Lawton and R. M. May, editors. Extinction rates. Oxford University Press, Oxford, UK. 
Tilman, D. 1994. Competition and biodiversity in spatially structured habitats. Ecology 75:685-700.

Tilman, D., and P. Kareiva. 1997. Spatial ecology: the role of space in population dynamics and interspecific interactions. Princeton University Press, Princeton, New Jersey, USA.
Valladares, G., L. Cagnolo, and A. Salvo. 2012. Forest fragmentation leads to food web contraction. Oikos 121:299-305.

Ying, Z., et al. 2014. Species coexistence in a lattice-structured habitat: Effects of species dispersal and interactions. Journal of Theoretical Biology 359:184-191.

\section{SUPPORTING INFORMATION}

Additional supporting information may be found in the online version of this article at http://onlinelibrary.wiley.com/doi/ 10.1002/ecy.1830/suppinfo 